\title{
SYNTHESIS OF DIBENZYLBUTANEDIOL LIGNANS AND THEIR ANTI-HIV, ANTI-HSV, ANTI-TUMOR ACTIVITIES
}

\author{
YA-MUXIA, ${ }^{*}$ WEN-HUI BI, YUAN-YUAN ZHANG
}

(College of Chemical Engineering, Qingdao University of Science and Technology, Shandong, Qingdao, 266042)

(Received: March 31, 2009 - Accepted: August 5, 2009)

\begin{abstract}
An efficient route to the synthesis of dibenzylbutanediol lignans and their analogues was reported. The syntheses were based on a strategy involving Stobbe condensation and alkylation reaction to give the skeleton of lignan, the resolution of $( \pm$ )-diacid with quinine, the transformation of functional groups to obtain seven dibenzylbutanediol lignans and one analogue. Among the synthesized lignans, four compounds were natural products. All dibenzylbutanediol lignans and their analogue synthesized were evaluated on HIV Tat transactivation in human epithelial cells, HSV-1 gene and human leukemic, liver, prostate, stomach, and breast cancer cell in vitro, but some compounds displayed weak activity against HIV, HSV and MDA-MB-435 human breast cancer cell.
\end{abstract}

Keywords: Dibenzylbutanediol lignan; Biological activity; Synthesis

\section{INTRODUCTION}

Lignans are a class of secondary plant metabolites produced by oxidative dimerization of two phenylpropanoid units. They are widely distributed in the plant kingdom and have been found in species belonging to more than seventy families. Lignans are found in roots, rhizomes, stems, leaves, seeds and fruits ${ }^{1,2}$. Lignans probably participate in plant development, and may play an important role in plant defense against various biological pathogens and pests. In addition to their purpose in nature, lignans also possess significant pharmacological activities, including antitumor, anti-inflammatory, immunosuppressive, cardiovascular, antioxidant and antiviral actions. From a chemical point of view, lignans show an enormous structural diversity, although their molecular backbone consists only of two phenylpropane units. Different families of lignans include the aryltetrahydronaphthalenes, typified by podophyllotoxin, the diverse family of dibenzocyclooctadienes, the dibenzylbutanediol lignans and so on ${ }^{3-6}$.

Dibenzylbutanediol lignans and their analogues are a class of lignans that have received considerable interest because of their biological and medicinal properties. The dibenzylbutanediol lignans seco-isolariciresinol and its metabolits enterodiol induced a significant decrease in the invasion (MDAMB231) through Matrigel ${ }^{7}$.Isotaxiresinol and its analogue (+)-taxiresinol from Taxus wallichiana were explored for anticancer properties. It was found that two lignans were active against colon adenocarcinoma cell lines in the MTT assay. However, isotaxiresinol was most active against the Caco-2 cell line in a clonogenic assay ${ }^{8}$. Kuepeli et al. investigated five dibenzylbutanediol lignans and the analogues for their anti-inflammatory activities, in which the lignans significantly inhibited carrageenan-induced hind paw edema in mice. The result showed that lariciresinol and isolariciresinol possess a potent in vitro inhibitory effect on the production of TNF- $\alpha$, a pro-inflammatory cytokine ${ }^{9}$. Other many dibenzylbutanediol lignans and their analogues have been shown to have a beneficial anti-virus effect ${ }^{10}$. These studies help to conclude that in future the dibenzylbutanediol lignans and their analogues may be an effective means of dealing with cancer, as well as providing antiviral and anti-inflammatory benefits.

Several strategies for the synthesis of dibenzylbutanediol lignans and their analogues have been reported ${ }^{11-15}$. Among them, the alkylation of $\beta$-substituted $\gamma$-butyrolactone with an appropriate benzylic halide is the most widely used ${ }^{16}$ Herein, we report an efficient route to the synthesis of dibenzylbutanediol lignans and their analogues. The syntheses were based on a strategy involving Stobbe condensation and alkylation reaction to give the skeleton of lignan, the transformation of functional groups to obtain target compound. All lignans synthesized were evaluated on HIV Tat transactivation in human epithelial cells, HSV-1 gene and human leukemic, liver, prostate, stomach, and breast cancer cell in vitro. We would like to point out structure-activity relationship could be established, and the mechanism of action was determined.

\section{EXPERIMENTAL}

Melting points were taken on Gallenkamp melting point apparatus and are uncorrected. Optical rotations were determined on a Perkin-Elmer 341 polarimeter. Infrared spectra were recorded on a Nicolet NEXUS 670 FT-IR.
The ${ }^{1} \mathrm{HNMR}$ and ${ }^{13} \mathrm{CNMR}$ spectra were recorded on Mercury Plus -300 $\mathrm{MHz}$ and Avance- $200 \mathrm{MHz}$ spectrometers. Mass spectra were recorded on a ZAB-HS spectrometer. HRMS were obtained on a Bruker Daltonics APEXII47e spectrometer. Flash column chromatography was performed on silica gel (200-300 mesh) and TLC inspections on silica gel $\mathrm{GF}_{254}$ plates.

Diethyl 2-(3',4'-methylenedioxybenzylidene)succinate $2 \mathrm{a}$. The piperonal 1a $(15.0 \mathrm{~g}, 100 \mathrm{mmol})$ and diethylsuccinate $(17.4 \mathrm{~g}, 100 \mathrm{mmol})$ were added to a solution of NaOEt $(13.6 \mathrm{~g}, 200 \mathrm{mmol})$ in EtOH $(200 \mathrm{ml})$. After refluxing for $4 \mathrm{hrs}$, ethanol was removed. The residue was cooled and acidified with $\mathrm{HCl}(5 \mathrm{~N})$. The mixture was extracted with EtOAc $(3 \times 80 \mathrm{ml})$. The EtOAc layer was then re-extracted with $\mathrm{NaHCO}_{3}$ saturated solution $(100 \mathrm{ml})$. The $\mathrm{NaHCO}_{3}$ extract was acidificated with $\mathrm{HCl}$ and the $\mathrm{pH}$ value was adjusted to 2 . Then the obtained an oily layer was again extracted with EtOAc $(3 \times 100$ $\mathrm{ml})$. The combined organic layer was dried over $\mathrm{MgSO}_{4}$ and concentrated in vacuo. This residue was then added to the mixture of $\mathrm{EtOH}(250 \mathrm{ml})$, benzene $(100 \mathrm{ml})$, and $\mathrm{H}_{2} \mathrm{SO}_{4}(2 \mathrm{ml})$, then refluxed in a Dean and Stark apparatus for $24 \mathrm{hrs}$ so that water removed. The reaction mixture was concentrated in vacuo and extracted with EtOAc $(200 \mathrm{ml})$, then washed with the $\mathrm{NaHCO}_{3}$ saturated solution $(3 \times 50 \mathrm{ml})$. The extract was dried over $\mathrm{MgSO}_{4}$ and concentrated in vacuo. Flash column chromatography of the residue afforded compound $2 \mathbf{a}$ as a yellow oil $(28.2 \mathrm{~g}, 92 \%)$. ${ }^{1} \mathrm{H}$ NMR $\left(200 \mathrm{MHz}, \mathrm{CDCl}_{3}\right): \delta 1.22(\mathrm{t}, J=7.3 \mathrm{~Hz}$, $3 \mathrm{H}), 1.29(\mathrm{t}, J=7.3 \mathrm{~Hz}, 3 \mathrm{H}), 3.51(\mathrm{~s}, 2 \mathrm{H}), 4.16$ (q, $J=7.3 \mathrm{~Hz}, 2 \mathrm{H}), 4.27$ (q, $J$ $=7.3 \mathrm{~Hz}, 2 \mathrm{H}), 5.96(\mathrm{~s}, 2 \mathrm{H}), 6.76-6.87(\mathrm{~m}, 3 \mathrm{H}), 7.76(\mathrm{~s}, 1 \mathrm{H}) ; \mathrm{EI}-\mathrm{MS}(\mathrm{m} / \mathrm{z}, \%)$ : $306\left(\mathrm{M}^{+}, 70\right), 261$ (20), 232 (34), 203 (52), 175 (59), 159 (100). Anal. Calcd for $\mathrm{C}_{16} \mathrm{H}_{18} \mathrm{O}_{6}: \mathrm{C}, 62.88 ; \mathrm{H}, 5.97$. Found: $\mathrm{C}, 62.74 ; \mathrm{H}, 5.92$.

Diethyl 2-(3',4'-dimethoxybenzylidene)succinate $\mathbf{2 b}$. Following the procedure described for the preparation of $\mathbf{2 a}$, and starting with the veratraldehyde $\mathbf{1 b}(16.6 \mathrm{~g}, 100 \mathrm{mmol})$, compound $\mathbf{2 b}$ was obtained as a yellow oil $(29.6$ g, 92\%). ${ }^{1} \mathrm{H}$ NMR (200 MHz, $\left.\mathrm{CDCl}_{3}\right): \delta 1.33$ (t, $\left.J=7.3 \mathrm{~Hz}, 3 \mathrm{H}\right), 1.26$ (t, $J=7.2$ $\mathrm{Hz}, 3 \mathrm{H}), 3.58(\mathrm{~s}, 2 \mathrm{H}), 3.87(\mathrm{~s}, 3 \mathrm{H}), 3.90(\mathrm{~s}, 3 \mathrm{H}), 4.21(\mathrm{q}, J=7.3 \mathrm{~Hz}, 2 \mathrm{H}), 4.27$ $(\mathrm{q}, J=7.3 \mathrm{~Hz}, 2 \mathrm{H}), 6.86-7.00(\mathrm{~m}, 3 \mathrm{H}), 7.84(\mathrm{~s}, 1 \mathrm{H}) ; \mathrm{EI}-\mathrm{MS}(\mathrm{m} / \mathrm{z}, \%): 322$ $\left(\mathrm{M}^{+}, 42\right), 276(14), 249(16), 175(100)$. Anal. Calcd for $\mathrm{C}_{17} \mathrm{H}_{22} \mathrm{O}_{6}: \mathrm{C}, 63.34 ; \mathrm{H}$, 6.88. Found: $\mathrm{C}, 63.31 ; \mathrm{H}, 6.83$.

Diethyl 2-(3',4'-methylenedioxybenzylidene)-3-(3',4', methylenedioxybenzyl) succinate 3a. To a well-stirred solution of compound $\mathbf{2 a}(24.5 \mathrm{~g}$, $80 \mathrm{mmol})$ in THF $(100 \mathrm{ml})$ was added dropwise a solution of LDA $(80 \mathrm{mmol}$, $2 \mathrm{M}$ in THF) in THF at $-78^{\circ} \mathrm{C}$ under nitrogen atmosphere. The mixture was stirred at this temperature for 20 mins, then 3,4- methylenedioxybenzyl bromide $(17.2 \mathrm{~g}, 80 \mathrm{mmol})$ in THF $(50 \mathrm{ml})$ was added. The mixture was stirred at $-78^{\circ} \mathrm{C}$ for $2 \mathrm{hrs}$. The mixture was quenched with $\mathrm{NH}_{4} \mathrm{Cl}$ saturated solution $(100$ $\mathrm{ml}$ ). After warmed to room temperature, the mixture was extracted with $\mathrm{CH}_{2} \mathrm{Cl}_{2}$ $(3 \times 80 \mathrm{ml})$ and the organic layer was dried over $\mathrm{MgSO}_{4}$ and concentrated in vacuo. Flash chromatography of the residue over silica gel gave compound $\mathbf{3 a}$ as a white crystal $(31.6 \mathrm{~g}, 90 \%)$. M.p. $58-59^{\circ} \mathrm{C}$. IR $\left(\mathrm{KBr} / \mathrm{cm}^{-1}\right): 3410,2981$, 1736, 1490, 1246, 1039, 930, 809, 770. ${ }^{1} \mathrm{H}$ NMR (200 MHz, CDCl $): \delta 1.26$ $(\mathrm{t}, J=7.2 \mathrm{~Hz}, 3 \mathrm{H}, \mathrm{CH}), 1.34\left(\mathrm{t}, J=7.2 \mathrm{~Hz}, 3 \mathrm{H}, \mathrm{CH}_{3}\right), 2.85(\mathrm{dd}, J=10.0,14.2$ $\mathrm{Hz}, 1 \mathrm{H}, \mathrm{H}-7$ ' ' $\alpha$ ), 3.34 (dd, $J=5.0,14.2 \mathrm{~Hz}, 1 \mathrm{H}, \mathrm{H}-7{ }^{3}$ ' $\beta$ ), 3.98 (dd, $J=5.0,10.0$ $\mathrm{Hz}, 1 \mathrm{H}, \mathrm{H}-3), 4.15-4.32\left(\mathrm{~m}, 4 \mathrm{H}, 2 \times \mathrm{CH}_{2} \mathrm{CH}_{3}\right), 5.88\left(\mathrm{~s}, 2 \mathrm{H}, \mathrm{OCH}_{2} \mathrm{O}\right), 5.97(\mathrm{~s}$, $\left.2 \mathrm{H}, \mathrm{OCH}_{2} \mathrm{O}\right), 6.35-6.73(\mathrm{~m}, 6 \mathrm{H}, \mathrm{ArH}), 7.66\left(\mathrm{~s}, 1 \mathrm{H}, \mathrm{H}-7\right.$ '). ${ }^{13} \mathrm{C}$ NMR $(50 \mathrm{MHz}$, $\left.\mathrm{CDCl}_{3}\right): \delta 14.4\left(2 \times \mathrm{CH}_{2} \mathrm{CH}_{3}\right), 36.0(\mathrm{C}-3), 45.8\left(\mathrm{C}-7{ }^{\prime \prime}\right), 61.2\left(2 \times \mathrm{OCH}_{2} \mathrm{CH}_{3}\right)$, $\left.100.9\left(\mathrm{OCH}_{2} \mathrm{O}\right), 101.4\left(\mathrm{OCH}_{2} \mathrm{O}\right), 108.1\left(\mathrm{C}-5^{\prime}\right), 108.4\left(\mathrm{C}-5^{\prime}{ }^{\prime}\right), 108.7\left(\mathrm{C}^{\prime}\right)^{\prime}\right)$, 
109.7 (C-2"'), 122.4 (C-6'), 122.7 (C-6"), 129.3 (C-1'), 130.1 (C-1'"), 133.2 (C-2), 142.5 (C-7'), 146.1(C-4'), 147.5 (C-4'), 147.8 (C-3', C-3''), 166.9 $(\mathrm{C}=\mathrm{O}), 172.9(\mathrm{C}=\mathrm{O})$. EI-MS $(\mathrm{m} / z, \%): 440\left(\mathrm{M}^{+}, 4\right), 395(1), 306(5), 231(40)$, 137 (100). HRMS caled for $\mathrm{C}_{24} \mathrm{H}_{25} \mathrm{O}_{8}\left(\mathrm{M}+\mathrm{H}^{+}\right)$: 441.1544. Found: 441.1538. Anal. Calcd for $\mathrm{C}_{24} \mathrm{H}_{24} \mathrm{O}_{8}: \mathrm{C}, 65.45 ; \mathrm{H}, 5.49$. Found: C, 65.41; H, 5.45.

Diethyl 2-(3',4'-dimethoxybenzylidene)-3-(3', ,4'-methylenedioxybenzyl)succinate $\mathbf{3 b}$. Following the procedure described for the preparation of $\mathbf{3 a}$, and starting with $\mathbf{2 b}(25.8 \mathrm{~g}, 80 \mathrm{mmol})$, compound $\mathbf{3 b}$ was obtained as a yellowish oil (31.7 g, 87\%). IR ( $\left.\mathrm{KBr} / \mathrm{cm}^{-1}\right): 2958,2839,1741,1517,918,810$, 760. ${ }^{1} \mathrm{H}$ NMR $\left(200 \mathrm{MHz}, \mathrm{CDCl}_{3}\right): \delta 1.26\left(\mathrm{t}, J=7.2 \mathrm{~Hz}, 3 \mathrm{H}, \mathrm{CH}_{3}\right), 1.35(\mathrm{t}, J=$ $\left.7.2 \mathrm{~Hz}, 3 \mathrm{H}, \mathrm{CH}_{3}\right), 2.91\left(\mathrm{dd}, J=9.8,14.2 \mathrm{~Hz}, 1 \mathrm{H}, \mathrm{H}-7{ }^{\prime}{ }^{\prime} \alpha\right), 3.34$ (dd, $J=5.0$, $14.2 \mathrm{~Hz}, 1 \mathrm{H}, \mathrm{H}-7$ ' $\beta), 3.78\left(\mathrm{~s}, 3 \mathrm{H}, \mathrm{OCH}_{3}\right), 3.88\left(\mathrm{~s}, 3 \mathrm{H}, \mathrm{OCH}_{3}\right), 4.10(\mathrm{dd}, J=5.0$, $9.8 \mathrm{~Hz}, 1 \mathrm{H}, \mathrm{H}-3), 4.18\left(\mathrm{q}, J=7.2 \mathrm{~Hz}, 2 \mathrm{H}, \mathrm{CH}_{2} \mathrm{CH}_{3}\right), 4.30(\mathrm{q}, J=7.2 \mathrm{~Hz}, 2 \mathrm{H}$, $\left.\mathrm{CH}_{2} \mathrm{CH}_{3}\right), 5.85\left(\mathrm{~s}, 2 \mathrm{H}, \mathrm{OCH}_{2} \mathrm{O}\right), 6.35-6.80(\mathrm{~m}, 6 \mathrm{H}, \mathrm{ArH}), 7.71\left(\mathrm{~s}, 1 \mathrm{H}, \mathrm{H}-7^{\prime}\right)$; ${ }^{13} \mathrm{C}$ NMR $\left(50 \mathrm{MHz}, \mathrm{CDCl}_{3}\right): \delta 14.1\left(\mathrm{CH}_{2} \mathrm{CH}_{3}\right), 14.2\left(\mathrm{CH}_{2} \mathrm{CH}_{3}\right), 35.7(\mathrm{C}-3), 45.5$ (C-7'”), $55.7\left(\mathrm{OCH}_{3}\right), 55.8\left(\mathrm{OCH}_{3}\right), 60.9\left(2 \times \mathrm{CH}_{2} \mathrm{CH}_{3}\right), 100.7\left(\mathrm{OCH}_{2} \mathrm{O}\right), 107.7$ (C-5'), 109.4 (C-5'), 110.7 (C-2'), 111.4 (C-2'”), 121.1 (C-6'), 122.0 (C-6'), 127.9 (C-1'), 129.5 (C-1'”), 132.9 (C-2), 142.3 (C-7'), 145.7 (C-4'), 147.2 (C-4'"), $148.6\left(\mathrm{C}-3^{\prime}\right), 149.1(\mathrm{C}-3$ '”), $166.7(\mathrm{C}=\mathrm{O}), 172.7(\mathrm{C}=\mathrm{O})$. EI-MS $(\mathrm{m} / \mathrm{z}$, \%): $456\left(\mathrm{M}^{+}, 3\right), 411$ (1), $382(1), 322$ (4), 247 (51), 137 (100). HRMS calcd for $\mathrm{C}_{25} \mathrm{H}_{29} \mathrm{O}_{8}\left(\mathrm{M}+\mathrm{H}^{+}\right): 457.1857$. Found: 457.1856. Anal. Calcd for $\mathrm{C}_{25} \mathrm{H}_{28} \mathrm{O}_{8}$ : C, 65.78; H, 6.18. Found: C, 65.72; H, 6.13.

(-)-2-(3',4'-methylenedioxybenzylidene)-3-(3', ,4' -methylenedioxybenzyl)succinic acid 4a. Diester 3a $(26.4 \mathrm{~g}, 60 \mathrm{mmol})$ was added to a solution of $20 \%$ aqueous $\mathrm{NaOH}(250 \mathrm{ml})$ and refluxed for $3 \mathrm{hrs}$. After cooled to room temperature, the mixture was washed with EtOAc $(3 \times 30 \mathrm{ml})$. After decolored with active carbon, the mixture was acidificated with $\mathrm{HCl}(2 \mathrm{~N})$, and obtained white solids. The crude product was crystallized from HOAc to give the $( \pm)$-diacid 4a. The ( \pm )-diacid 4a and (-)-quinine (38.9 g, $120 \mathrm{mmol})$ in ethanol (120 $\mathrm{ml}$ ) was refluxed for $1 \mathrm{hrs}$. The reaction mixture was allowed to cool to room temperature slowly, fine white crystals were obtained. Two recrystallizations from ethanol was added to a solution of $\mathrm{HCl}(2 \mathrm{~N}, 100 \mathrm{ml})$ and stirred for $1 \mathrm{hrs}$. The mixture was extracted with EtOAc $(3 \times 80 \mathrm{ml})$, and the extract was dried over $\mathrm{MgSO}_{4}$ and evaporated. The white solids were recrystallized in EtOAc to yield the (-)-diacid 4 a as a white crystal $(10.1$ g, $44 \%)$. M.p. $98-99^{\circ} \mathrm{C}$. $[\alpha]^{16}$ -95.3 (c 1.0, EtOH). IR ( $\left.\mathrm{KBr} / \mathrm{cm}^{-1}\right): 3385,2898,1703,1498,1242,1040,928$, $813,620 .{ }^{1}{ }^{H}$ NMR $\left(200 \mathrm{MHz}\right.$, DMSO- $\left.d_{6}\right): \delta 2.85(\mathrm{dd}, J=10.2,13.8 \mathrm{~Hz}, 1 \mathrm{H}$, H-7' ' $\alpha$ ), 3.25 (dd, $J=4.4,13.8 \mathrm{~Hz}, 1 \mathrm{H}, \mathrm{H}-7$ '' $\beta$ ), 3.93 (dd, $J=4.4,10.2 \mathrm{~Hz}$, $1 \mathrm{H}, \mathrm{H}-3), 5.92\left(\mathrm{~d}, J=7.6 \mathrm{~Hz}, 2 \mathrm{H}, \mathrm{OCH}_{2} \mathrm{O}\right), 6.04\left(\mathrm{~s}, 2 \mathrm{H}, \mathrm{OCH}_{2} \mathrm{O}\right), 6.37-6.90$ (m, 6H, ArH), 7.53 (s, 1H, H-7'). EI-MS ( $m / z, \%): 384\left(\mathrm{M}^{+}, 1\right), 366(1), 244$ (1), 203 (3), 159 (2), 135 (100). Anal. Calcd for $\mathrm{C}_{20} \mathrm{H}_{16} \mathrm{O}_{8}$ : C, 62.50; H, 4.20. Found: C, 62.46; H, 4.17 .

The white solids from concentrating the mother liquors were recrystallized twice in methanol and water to yield the (+)-diacid $\mathbf{4} \mathbf{a}^{\mathbf{\prime}}$ as a white crystal $(9.0$ g, 39\%). M.p. $96-97^{\circ} \mathrm{C} .[\alpha]_{\mathrm{D}}{ }^{16}+94.8$ (c 0.8, EtOH). ${ }^{1} \mathrm{H}$ NMR, IR and MS of $4 \mathbf{a}^{\prime}$ are in agreement with $\mathbf{4 a}$

(-)-2-(3',4'-Dimethoxybenzylidene)-3-(3', 4''-methylenedioxybenzyl) succinic acid $\mathbf{4 b}$. Following the procedure described for the preparation of $\mathbf{4 a}$, and starting with the diester $\mathbf{3 b}(27.4 \mathrm{~g}, 60 \mathrm{mmol})$, (-)-diacid $\mathbf{4 b}$ was obtained as a white crystal $(10.8 \mathrm{~g}, 45 \%)$. M.p. $159-160^{\circ} \mathrm{C}$. $[\alpha]_{\mathrm{D}}{ }^{16}-143.2$ (c $\left.0.7, \mathrm{EtOH}\right)$. IR $\left(\mathrm{KBr} / \mathrm{cm}^{-1}\right): 3522,2943,1714,1516,1255,1142,925 .{ }^{1} \mathrm{H}$ NMR $(200 \mathrm{MHz}$, DMSO- $d_{6}$ ): $\delta 2.83$ (dd, $J=10.2,14.0 \mathrm{~Hz}, 1 \mathrm{H}, \mathrm{H}-7$ ' ' $\alpha$ ), 3.18 (dd, $J=4.8,14.0$ $\mathrm{Hz}, 1 \mathrm{H}, \mathrm{H}-7$ ' $\beta), 3.64\left(\mathrm{~s}, 3 \mathrm{H}, \mathrm{OCH}_{3}\right), 3.73\left(\mathrm{~s}, 3 \mathrm{H}, \mathrm{OCH}_{3}\right), 3.95$ (dd, $J=4.8$, $10.2 \mathrm{~Hz}, 1 \mathrm{H}, \mathrm{H}-3), 5.87\left(\mathrm{~d}, J=8.2 \mathrm{~Hz}, 2 \mathrm{H}, \mathrm{OCH}_{2} \mathrm{O}\right), 6.35-6.89(\mathrm{~m}, 6 \mathrm{H}, \mathrm{ArH})$, $7.52\left(\mathrm{~s}, 1 \mathrm{H}, \mathrm{H}-7^{\prime}\right)$. EI-MS $(\mathrm{m} / z, \%): 400\left(\mathrm{M}^{+}, 1\right), 382(17), 260(5), 219(12)$, 175 (26), 135 (100). HRMS calcd for $\mathrm{C}_{21} \mathrm{H}_{21} \mathrm{O}_{8}\left(\mathrm{M}+\mathrm{H}^{+}\right)$: 401.1231. Found: 401.1239. Anal. Calcd for $\mathrm{C}_{21} \mathrm{H}_{20} \mathrm{O}_{8}: \mathrm{C}, 63.00 ; \mathrm{H}, 5.03$. Found: $\mathrm{C}, 62.96 ; \mathrm{H}$, 5.01 .

$(+)$-diacid $\mathbf{4} \mathbf{b}^{\prime}$ was obtained as a white crystal $(9.2 \mathrm{~g}, 38 \%)$. M.p. $89-91{ }^{\circ} \mathrm{C}$. $[\alpha]_{\mathrm{D}}{ }^{16}+142.6(\mathrm{c} 0.6, \mathrm{EtOH}) .{ }^{1} \mathrm{H}$ NMR, IR and MS of $\mathbf{4} \mathbf{b}^{\prime}$ are in agreement with 4b.

(-)-Diethyl 2-(3',4'-methylenedioxybenzylidene)-3-(3',4',-methylenedioxybenzyl)succinate $5 \mathbf{a}$. To the $151 \mathrm{ml}$ mixture of $\mathrm{EtOH}$ : benzene : $\mathrm{H}_{2} \mathrm{SO}_{4}$ $(100: 50: 1)$ was added $4 \mathrm{a}(7.7 \mathrm{~g}, 20 \mathrm{mmol})$, refluxed in a Dean and Stark apparatus for $36 \mathrm{hrs}$ so that water removed. The reaction mixture was concentrated in vacuo and extracted with EtOAc $(100 \mathrm{ml})$, and then neutralized with the $\mathrm{NaHCO}_{3}$ saturated solution $(3 \times 30 \mathrm{ml})$. The extract was dried over $\mathrm{MgSO}_{4}$ and concentrated in vacuo. Flash column chromatography of the residue gave $(-)$-diester 5a as a colorless oil $(8.0 \mathrm{~g}, 91 \%)$. $[\alpha]_{\mathrm{D}}{ }^{16}-68.4\left(\mathrm{c} 1.0, \mathrm{CHCl}_{3}\right) .{ }^{1} \mathrm{H}$ NMR, IR, MS and HRMS of $\mathbf{5 a}$ are in agreement with $\mathbf{3 a}$.

(-)-Diethyl 2-(3',4'-Dimethoxybenzylidene)-3-(3',,4',-methylenedioxybenzyl)succinate $\mathbf{5 b}$. Following the procedure described for the preparation of $\mathbf{5 a}$, and starting with the diester $\mathbf{4 b}(8.0 \mathrm{~g}, 20 \mathrm{mmol})$, diacid $\mathbf{5 b}$ was obtained as a colorless oil $(8.2 \mathrm{~g}, 90 \%) \cdot[\alpha]_{\mathrm{D}}{ }^{16}-170.1$ (c 1.0, $\left.\mathrm{CHCl}_{3}\right) .{ }^{1} \mathrm{H} \mathrm{NMR}$, IR, MS and HRMS of $\mathbf{5 b}$ are in agreement with $\mathbf{3 b}$

(-)-2-(3',4'-methylenedioxybenzylidene)-3-(3',,4',-methylenedioxybenzyl)butane-1,4-diol 6a. $\mathrm{AlCl}_{3}(0.32 \mathrm{~g}, 2.4 \mathrm{mmol})$ added to a stirred suspension of $\mathrm{LiAlH}_{4}(0.32 \mathrm{~g}, 8 \mathrm{mmol})$. The mixture was stirred for 20 mins. Then (-)-diester 5a (3.72 g, $8 \mathrm{mmol})$ was added to the solution and the reaction mixture was stirred for $10 \mathrm{hrs}$. The reaction was quenched by ice water and filtered. The filtrate was dried over $\mathrm{MgSO}_{4}$ and concentrated in vacuo. Flash column chromatography of the residue gave $(-)-6 \mathbf{a}\left(2.5 \mathrm{~g}^{\circ} \mathrm{C} 87 \%\right)$. White crystals. M.p. $125-126^{\circ} \mathrm{C}$. $[\alpha]_{\mathrm{D}}{ }^{16}-27.6\left(\mathrm{c} 0.4, \mathrm{CHCl}_{3}\right)$. IR $\left(\mathrm{KBr} / \mathrm{cm}^{-1}\right): 3375$, $1600,1490,920 .{ }^{1} \mathrm{H}$ NMR $\left(300 \mathrm{MHz}, \mathrm{CDCl}_{3}\right): \delta 2.46-2.82\left(\mathrm{~m}, 2 \mathrm{H}, \mathrm{ArCH}_{2}\right)$, $3.38-3.63(\mathrm{~m}, 1 \mathrm{H}, \mathrm{H}-3), 4.02-4.48(\mathrm{~m}, 4 \mathrm{H}, \mathrm{H}-1, \mathrm{H}-4), 5.90\left(\mathrm{~s}, 2 \mathrm{H}, \mathrm{OCH}_{2} \mathrm{O}\right)$, $5.97\left(\mathrm{~s}, 2 \mathrm{H}, \mathrm{OCH}_{2} \mathrm{O}\right), 6.54-6.82(\mathrm{~m}, 6 \mathrm{H}, \mathrm{ArH}), 7.61(\mathrm{~s}, 1 \mathrm{H}, \mathrm{ArCH}=\mathrm{C}) ;{ }^{2}{ }^{13} \mathrm{C}$ NMR (50 MHz, CDCl ${ }_{3}$ ): 35.8 (C-3), 45.6 (C-7'), 62.3 (C-4), 67.1 (C-1), $100.8\left(\mathrm{OCH}_{2} \mathrm{O}\right), 108.2$ (C-5'), 109.1 (C-5') 109.9 (C-2'), 110.4 (C-2'”), 121.4 (C-6'), 122.3 (C-6'), 127.9 (C-1'), 130.6 (C-1''), 132.4 (C-2), 141.3 (C-7'), 145.7 (C-4'), 147.8 (C-4'”), 149.1 (C-3'”), 149.2 (C-3'); EI-MS (m/z, \%): $356\left(\mathrm{M}^{+}, 2\right), 338(3), 221(5), 203(16), 135$ (100). HRMS calcd for $\mathrm{C}_{20} \mathrm{H}_{24} \mathrm{NO}_{6}$ $\left(\mathrm{M}+\mathrm{NH}_{4}^{+}\right): 374.1599$. Found: 374.1592. Anal. Calcd for $\mathrm{C}_{20} \mathrm{H}_{20} \mathrm{O}_{6}: \mathrm{C}, 67.41 ; \mathrm{H}$, 5.66. Found: $\mathrm{C}, 67.38 ; \mathrm{H}, 5.63$.

(+)-2-(3',4'-Dimethoxybenzylidene)-3-(3',4''-methylenedioxybenzyl) butane-1,4-diol 6b. Following the procedure described for the preparation of $\mathbf{6 a}$, and starting with the (-)-diester $\mathbf{5 b}(3.7 \mathrm{~g}, 8 \mathrm{mmol})$, the $\mathbf{6 b}$ was obtained as a colorless oil $(2.6 \mathrm{~g}, 85 \%)$. $[\alpha]^{16}+14.0\left(\mathrm{c} 2.9, \mathrm{CH}_{3} \mathrm{COCH}_{3}\right)$. IR $\left(\mathrm{KBr} / \mathrm{cm}^{-1}\right):=$ $3382,1600,1493,920 .{ }^{1} \mathrm{H}$ NMR $\left(300 \mathrm{MHz}, \mathrm{CDCl}_{3}\right): \delta 2.45-2.80(\mathrm{~m}, 2 \mathrm{H}$, $\left.\mathrm{ArCH}_{2}\right), 3.42-3.67(\mathrm{~m}, 1 \mathrm{H}, \mathrm{H}-3), 3.77\left(\mathrm{~s}, 3 \mathrm{H}, \mathrm{OCH}_{3}\right), 3.89\left(\mathrm{~s}, 3 \mathrm{H}, \mathrm{OCH}_{3}\right)$, 4.05-4.46 (m, 4H, H-1, H-4), $5.92\left(\mathrm{~s}, 2 \mathrm{H}, \mathrm{OCH}_{2} \mathrm{O}\right), 6.52-6.87$ (m, 6H, ArH), $7.67(\mathrm{~s}, 1 \mathrm{H}, \mathrm{ArCH}=\mathrm{C}) ;{ }^{13} \mathrm{C}$ NMR (50 MHz, $\left.\mathrm{CDCl}_{3}\right): 35.2(\mathrm{C}-3), 45.1(\mathrm{C}-7$ ') $55.6\left(\mathrm{OCH}_{3}\right), 55.8\left(\mathrm{OCH}_{3}\right), 62.0(\mathrm{C}-4), 66.8(\mathrm{C}-1), 100.8\left(\mathrm{OCH}_{2} \mathrm{O}\right), 107.2$ (C-5'), 109.4 (C-5'), 109.8 (C-2'), 111.3 (C-2'”), 120.9 (C-6'), 122.0 (C-6'), 128.2 (C-1'), 129.8 (C-1'”), 133.8 (C-2), 142.1 (C-7'), 144.9 (C-4'), 147.2 (C-4"'), 150.2 (C-3"'), 150.6 (C-3"'); EI-MS ( $/ 2 / z, \%): 372\left(\mathrm{M}^{+}, 12\right), 354$ (8), 203 (7), 135 (100). HRMS calcd for $\mathrm{C}_{21} \mathrm{H}_{29} \mathrm{NO}_{6}\left(\mathrm{M}+\mathrm{NH}_{4}^{+}\right)$: 390.1912. Found: 390.1905. Anal. Calcd for $\mathrm{C}_{21} \mathrm{H}_{24} \mathrm{O}_{6}: \mathrm{C}, 67.73 ; \mathrm{H}, 6.50$. Found: $\mathrm{C}, 67.69 ; \mathrm{H}$, 6.47 .

(-)-Dihydrocubebin $7 \mathrm{a}$ and meso-2,3-Bis(3',4'-methylenedioxybenzyl) butane-1,4-diol 8a. (-)-Diester 5a (3.6 g, $8 \mathrm{mmol})$ in ethyl acetate $(100 \mathrm{ml})$ was stirred under hydrogen atmosphere for $12 \mathrm{hrs}$ in the presence of $10 \% \mathrm{Pd} / \mathrm{C}$ $(0.35 \mathrm{~g})$. The reaction mixture was filtered through a pad of Celite, and the solvent was removed in vacuo to give a white solid. The solid was dissolved in dry THF (40 ml) and added to a stirred suspension of $\mathrm{LiAlH}_{4}(0.7 \mathrm{~g}, 18$ $\mathrm{mmol}$ ). The mixture was stirred for $10 \mathrm{hrs}$. Then the reaction was quenched by ice water and filtered. The filtrate was dried over $\mathrm{MgSO}_{4}$ and concentrated in vacuo. Flash column chromatography of the residue gave threo-(-)-7a $(1.34 \mathrm{~g})$ and erythro-8a (1.31 g) . (-)-Dihydrocubebin (7a). Yield 47\%. White crystals. M.p. $112-113^{\circ} \mathrm{C}$. $[\alpha]^{16}-41.9$ (c $\left.0.8, \mathrm{CHCl}_{3}\right)$. IR $\left(\mathrm{KBr} / \mathrm{cm}^{-1}\right)$ : 3382,2921 , 1514, 1242, 1032, 928, 809, 764. ${ }^{1} \mathrm{H}$ NMR $\left(200 \mathrm{MHz}, \mathrm{CDCl}_{3}\right): \delta 1.73-1.84$ $(\mathrm{m}, 2 \mathrm{H}, \mathrm{H}-2, \mathrm{H}-3), 2.55-2.81\left(\mathrm{~m}, 4 \mathrm{H}, 2 \times \mathrm{ArCH}_{2}\right), 3.48(\mathrm{~d}, J=11.2 \mathrm{~Hz}, 2 \mathrm{H}$, $\left.\mathrm{CH}_{2} \mathrm{OH}\right), 3.72(\mathrm{~s}, 2 \mathrm{H}, 2 \times \mathrm{OH}), 3.74(\mathrm{~d}, J=11.2 \mathrm{~Hz}, 2 \mathrm{H}, \mathrm{CH} / \mathrm{OH}), 5.91(\mathrm{~s}, 4 \mathrm{H}$, $\left.2 \times \mathrm{OCH}_{2} \mathrm{O}\right), 6.58-6.73(\mathrm{~m}, 6 \mathrm{H}, \mathrm{ArH}) ;{ }^{13} \mathrm{C}$ NMR $\left(100 \mathrm{MHz}, \mathrm{CDCl}_{3}\right): \delta 35.8$

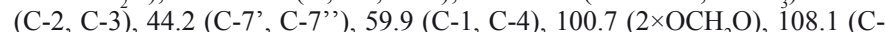
5', C-5"'), 109.2 (C-2', C-2'”), 121.8 (C-6', C-6"'), 134.3 (C-1', C-1'"), 145.6 (C-4', C-4'”), 147.5 (C-3', C-3"'). EI-MS (m/z, \%): $358\left(\mathrm{M}^{+}, 2\right), 340$ (0.1), 204 (0.3), 161 (3), 135 (100). HRMS calcd for $\mathrm{C}_{20} \mathrm{H}_{26} \mathrm{NO}_{6}\left(\mathrm{M}+\mathrm{NH}_{4}^{+}\right): 376.1755$ Found: 376.1760. Anal. Calcd for $\mathrm{C}_{20} \mathrm{H}_{22} \mathrm{O}_{6}:{ }^{20} \mathrm{C}, 67.03 ; \mathrm{H}, 6.19$. Found: $\mathrm{C}$, 66.98; $\mathrm{H}, 6.15$. The spectral data were in agreement with the literature ${ }^{17}$. meso-2,3-Bis(3',4'-methylenedioxybenzyl)butane-1,4-diol (8a). Yield $46 \%$. Colorless oil. IR $\left(\mathrm{KBr} / \mathrm{cm}^{-1}\right): 3293,2920,1488,1246,1037,928,811,731 .{ }^{1} \mathrm{H}$ NMR (200 MHz, $\left.\mathrm{CDCl}_{3}\right): \delta 1.91-2.05(\mathrm{~m}, 2 \mathrm{H}, \mathrm{H}-2, \mathrm{H}-3), 2.49-2.63(\mathrm{~m}, 4 \mathrm{H}$, $\left.2 \times \mathrm{ArCH}_{2}\right), 3.45-3.61\left(\mathrm{~m}, 4 \mathrm{H}, 2 \times \mathrm{CH}_{\mathrm{OH}}\right), 3.71(\mathrm{~s}, 2 \mathrm{H}, 2 \times \mathrm{OH}), 5.92(\mathrm{~s}$, $\left.4 \mathrm{H}, 2 \times \mathrm{OCH}_{2} \mathrm{O}\right), 6.61-6.76(\mathrm{~m}, 6 \mathrm{H}, \mathrm{ArH}) .{ }^{13} \mathrm{C} \mathrm{NMR}\left(50 \mathrm{MHz}, \mathrm{CDCl}_{3}\right): \delta 33.4$ (C-2, C-3), 45.2 (C-7', C-7'), 62.9 (C-1, C-4), $100.8\left(2 \times \mathrm{OCH}_{2} \mathrm{O}\right), 108.1$ (C5', C-5'), 109.2 (C-2', C-2'”), 121.8 (C-6', C-6'), 134.1 (C-1', C-1'”), 145.8 (C-4', C-4"'), 147.6 (C-3', C-3"'). EI-MS (m/z, \%): $358\left(\mathrm{M}^{+}, 3\right), 340(0.3), 204$ (0.8), 161 (4), 135 (100). HRMS calcd for $\mathrm{C}_{20} \mathrm{H}_{26} \mathrm{NO}_{6}\left(\mathrm{M}+\mathrm{NH}_{4}^{+}\right)$: 376.1755 Found: 376.1760. Anal. Calcd for $\mathrm{C}_{20} \mathrm{H}_{22} \mathrm{O}_{6}:{ }^{20} \mathrm{C}, 67.03$; H, 6.19. Found: $\mathrm{C}$, 66.98; H, 6.15.

(-)-Dihydro-3', 4'-dimethoxy-3", 4'-demethylenedioxycubebin 7b and (-)-2,3-Desmethoxy seco-isolintetralin 8b. Following the procedure described for the preparation of $\mathbf{7 a}$ and $\mathbf{8 a}$, and starting with the diester $\mathbf{5 b}(3.6$ g, $8 \mathrm{mmol})$, the $\mathbf{7 b}(1.3 \mathrm{~g})$ and $\mathbf{8 b}(1.45 \mathrm{~g})$ were obtained. (-)-Dihydro-3', $\mathbf{4}^{\prime}-$ dimethoxy-3",4"'-demethylenedioxycubebin (7b). Yield 44\%. Colorless oil. $[\alpha]_{\mathrm{D}}{ }^{16}-36.8\left(\mathrm{c} 0.5, \mathrm{CHCl}_{3}\right)$. IR $\left(\mathrm{KBr} / \mathrm{cm}^{-1}\right): 3374,1593,1515,1488,1442,928$. 
${ }^{1} \mathrm{H}$ NMR $\left(200 \mathrm{MHz}, \mathrm{CDCl}_{3}\right): \delta 1.73-1.92(\mathrm{~m}, 2 \mathrm{H}, \mathrm{H}-2, \mathrm{H}-3), 2.60-2.80(\mathrm{~m}, 4 \mathrm{H}$, $2 \times \mathrm{H}-7$ ', $2 \times \mathrm{H}-7 "), 3.50(\mathrm{~d}, 2 \mathrm{H}, J=11.6 \mathrm{~Hz}, \mathrm{CH} 2 \mathrm{OH}), 3.56(\mathrm{~s}, 2 \mathrm{H}, 2 \times \mathrm{OH})$, $3.80(\mathrm{~d}, 2 \mathrm{H}, J=11.6 \mathrm{~Hz}, \mathrm{CH} \mathrm{OH}), 3.82\left(\mathrm{~s}, 3 \mathrm{H}, \mathrm{OCH}_{3}\right), 3.84\left(\mathrm{~s}, 3 \mathrm{H}, \mathrm{OCH}_{3}\right)$, $5.90\left(\mathrm{~s}, 2 \mathrm{H}, \mathrm{OCH}_{2} \mathrm{O}\right), 6.57-6.80(\mathrm{~m}, 6 \mathrm{H}, \mathrm{ArH}) .{ }^{13} \mathrm{C} \mathrm{NMR}\left(100 \mathrm{MHz}, \mathrm{CDCl}_{3}\right)$ :

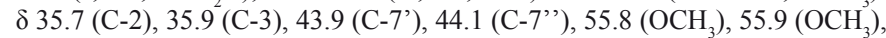
60.2 (C-1), 60.3 (C-4), $100.7\left(\mathrm{OCH}_{2} \mathrm{O}\right), 108.0$ (C-5'), 109.3 (C-5"), 111.2 (C-2'), 112.1 (C-2'), 121.0 (C-6'), 121.8 (C-6'), 133.1 (C-1'), 134.3 (C-1'), 145.7 (C-4'), 147.3 (C-4'”), 147.5 (C-3'), 148.8 (C-3'”). EI-MS ( $m / z, \%): 374$ $\left(\mathrm{M}^{+}, 4\right), 356(0.4), 220(3), 203(3), 151$ (100). HRMS calcd for $\mathrm{C}_{21} \mathrm{H}_{30} \mathrm{NO}$ $\left(\mathrm{M}+\mathrm{NH}_{4}^{+}\right)$: 392.2068. Found: 392.2063. Anal. Calcd for $\mathrm{C}_{21} \mathrm{H}_{26} \mathrm{O}_{6}: \mathrm{C}, 67.36$; $\mathrm{H}, 7.00$. Found: $\mathrm{C}, 67.31 ; \mathrm{H}, 6.97$. The spectral data were in agreement with the literature ${ }^{18}$. (-)-2,3-Desmethoxy seco-isolintetralin (8b). Yield 48\%. Colorless oil. $[\alpha]_{\mathrm{D}}{ }^{16}-1.7\left(\mathrm{c} 0.3, \mathrm{CHCl}_{3}\right)$. IR $\left(\mathrm{KBr} / \mathrm{cm}^{-1}\right): 3365,2919,1514,1241$, $1032,727,643 .{ }^{1} \mathrm{H}$ NMR $\left(200 \mathrm{MHz}, \mathrm{CDCl}_{3}\right): \delta 1.71-1.93(\mathrm{~m}, 2 \mathrm{H}, \mathrm{H}-2, \mathrm{H}-3)$, 2.56-2.82 (m, 4H, $\left.2 \times \mathrm{H}^{-7}, 2 \times \mathrm{H}-7 "\right), 3.50(\mathrm{~d}, 2 \mathrm{H}, J=11.0 \mathrm{~Hz}, \mathrm{CH}-\mathrm{OH}), 3.75$ (d, $\left.2 \mathrm{H}, J=11.0 \mathrm{~Hz}, \mathrm{CH}_{2} \mathrm{OH}\right), 3.81\left(\mathrm{~s}, 3 \mathrm{H}, \mathrm{OCH}_{3}\right), 3.83\left(\mathrm{~s}, 3 \mathrm{H}, \mathrm{OCH}_{3}\right), 3.95(\mathrm{~s}$, $2 \mathrm{H}, 2 \times \mathrm{OH}), 5.89\left(\mathrm{~s}, 2 \mathrm{H}, \mathrm{OCH}_{2} \mathrm{O}\right), 6.56-6.78(\mathrm{~m}, 6 \mathrm{H}, \mathrm{ArH}) .{ }^{13} \mathrm{C} \mathrm{NMR}(100$ $\left.\mathrm{MHz}, \mathrm{CDCl}_{3}\right)$ : $\delta 33.1$ (C-2), 33.4 (C-3), 45.0 (C-7'), 45.2 (C-7' '), $55.8\left(\mathrm{OCH}_{3}\right)$, $55.9\left(\mathrm{OCH}_{3}\right), 62.9(\mathrm{C}-1), 63.0(\mathrm{C}-4), 100.8\left(\mathrm{OCH}_{2} \mathrm{O}\right), 108.1\left(\mathrm{C}-5^{\prime}\right), 109.3$ (C-5'), 111.2 (C-2'), 112.1 (C-2'), 121.0 (C-6'), 121.8 (C-6'), 133.0 (C-1'), 134.2 (C-1"'), 145.8 (C-4'), 147.3 (C-4"'), 147.6 (C-3'), 148.8 (C-3'). EI-MS ( $\mathrm{m} / \mathrm{z}, \%): 374\left(\mathrm{M}^{+}, 4.7\right), 356(0.23), 220$ (1.8), 203 (2.5), 151 (100). HRMS calcd for $\mathrm{C}_{21} \mathrm{H}_{30} \mathrm{NO}_{6}\left(\mathrm{M}+\mathrm{NH}_{4}^{+}\right)$: 392.2068. Found: 392.2063. Anal. Calcd for $\mathrm{C}_{21} \mathrm{H}_{26} \mathrm{O}_{6}: \mathrm{C}, 67.36 ; \mathrm{H}, 7.00$. Found: $\mathrm{C}, 67.31 ; \mathrm{H}, 6.97$. The spectral data were in agreement with the literature ${ }^{19}$.

$( \pm)$-Dihydrosesamin 9. DDQ $(0.34 \mathrm{~g}, 1.5 \mathrm{mmol})$ added to a solution of dihydrocubebin $7 \mathbf{a}(0.36 \mathrm{~g}, 1 \mathrm{mmol})$ in glacial acetic acid. The mixture stirred for $5 \mathrm{hrs}$. The reaction mixture was poured onto crushed ice and extracted with EtOAc $(80 \mathrm{ml})$. The organic layer was washed with the $\mathrm{NaHSO}_{3}$ saturated solution $(3 \times 30 \mathrm{ml})$ and the $\mathrm{NaHCO}_{3}$ saturated solution $(3 \times 30 \mathrm{ml})$. The extract was dried over $\mathrm{MgSO}_{4}$ and concentrated in vacuo. Flash column chromatography of the residue gave ( \pm )-dihydrosesamin 9 as a colorless oil $(0.15 \mathrm{~g}, 42 \%)$. IR $\left(\mathrm{KBr} / \mathrm{cm}^{-1}\right): 3431,2934,1592,1514,1466,1230,1022 .{ }^{1} \mathrm{H}$ NMR (300 MHz, CDCl $)$ ): $\delta 1.67(\mathrm{~s}, 1 \mathrm{H}, \mathrm{OH}), 2.25-2.47(\mathrm{~m}, 1 \mathrm{H}, \mathrm{H}-3), 2.51$ (dd, $1 \mathrm{H}, J=10.3,13.1 \mathrm{~Hz}, \mathrm{H}-6 \alpha), 2.55-2.73(\mathrm{~m}, 1 \mathrm{H}, \mathrm{H}-4), 2.85$ (dd, $1 \mathrm{H}, J=$ 4.9, $13.1 \mathrm{~Hz}, \mathrm{H}-6 \beta$ ), 3.72 (dd, $1 \mathrm{H}, J=6.3,8.5 \mathrm{~Hz}, \mathrm{H}-5 \alpha$ ), 3.74 (dd, $1 \mathrm{H}, J=6.6$, $10.8 \mathrm{~Hz}, \mathrm{H}-7 \alpha), 3.86(\mathrm{dd}, 1 \mathrm{H}, J=6.9,10.7 \mathrm{~Hz}, \mathrm{H}-7 \beta), 4.03$ (dd, $1 \mathrm{H}, J=6.5$, $8.5 \mathrm{~Hz}, \mathrm{H}-5 \beta), 4.77(\mathrm{~d}, 1 \mathrm{H}, J=6.2 \mathrm{~Hz}, \mathrm{H}-2), 5.92\left(\mathrm{~s}, 2 \mathrm{H}, \mathrm{OCH}_{2} \mathrm{O}\right), 5.93(\mathrm{~s}, 2 \mathrm{H}$, $\left.\mathrm{OCH}_{2} \mathrm{O}\right), 6.60-6.82(\mathrm{~m}, 6 \mathrm{H}, \mathrm{ArH}) .{ }^{13} \mathrm{C}$ NMR $\left(75 \mathrm{MHz}, \mathrm{CDCl}_{3}\right): \delta 33.4(\mathrm{C}-6)$, 42.7 (C-4), 52.9 (C-3), 60.7 (C-7), $73.3(\mathrm{C}-5), 82.7$ (C-2), $101.0\left(\mathrm{OCH}_{2} \mathrm{O}\right)$ $101.1\left(\mathrm{OCH}_{2} \mathrm{O}\right), 106.6\left(\mathrm{C}-5^{\prime}\right), 108.2\left(\mathrm{C}-5^{\prime \prime}\right), 108.7\left(\mathrm{C}-2{ }^{\prime}\right), 109.3$ (C-2') 119.3 (C-6'), 121.4 (C-6'), 134.1 (C-1'), 137.4 (C-1'”), 146.1 (C-4'), 147.2 (C-4') 147.8 (C-3'), 148.2 (C-3'). EI-MS (m/z, \%): $356\left(\mathrm{M}^{+}, 12\right), 217$ (16), $192(15)$, 149 (21), 135 (100). HRMS calcd for $\mathrm{C}_{20} \mathrm{H}_{24} \mathrm{NO}_{6}\left(\mathrm{M}+\mathrm{NH}_{4}^{+}\right): 374.1599$. Found: 374.1603. Anal. Caled for $\mathrm{C}_{20} \mathrm{H}_{20} \mathrm{O}_{6}: \mathrm{C}^{20} 67.41 ; \mathrm{H}, 5.66$. Found: $\mathrm{C}, 67.37 ; \mathrm{H}$, 5.63. The spectral data were in agreement with the literature ${ }^{20}$.

( \pm )-Dibenzocyclooctadiene diol 10. To a mixture dihydrocubebin $7 \mathbf{a}(0.53$ $\mathrm{g}, 1.5 \mathrm{mmol})$ and DDQ $(0.79 \mathrm{~g}, 3.5 \mathrm{mmol})$ was added freshly distilled TFA (12 $\mathrm{ml})$. The mixture stirred for $2 \mathrm{hrs}$. The reaction mixture was poured onto crushed ice and extracted with EtOAc $(60 \mathrm{ml})$. The organic layer was washed with the $\mathrm{NaHSO}_{3}$ saturated solution $(3 \times 30 \mathrm{ml})$ and the $\mathrm{NaHCO}_{3}$ saturated solution $(3 \times 30 \mathrm{ml})$. The extract was dried over $\mathrm{MgSO}_{4}$ and concentrated in vacuo. Flash column chromatography of the residue gave $( \pm)$-Dibenzocyclooctadiene diol 10 a white crystal $(0.28 \mathrm{~g}, 52 \%)$. M.p. $270-272^{\circ} \mathrm{C}$. IR $\left(\mathrm{KBr} / \mathrm{cm}^{-1}\right): 3428,2936$, $1613,1512,1452,1207 .{ }^{1} \mathrm{H}$ NMR $\left(300 \mathrm{MHz}, \mathrm{CDCl}_{3}\right): \delta 1.32-1.56(\mathrm{~m}, 2 \mathrm{H}$, H-2, H-3), 2.07 (dd, 2H, $J=10.2,13.0 \mathrm{~Hz}, \mathrm{H}-7$ ' $\alpha, \mathrm{H}-7$ ' ' $\alpha$ ), 2.73 (dd, $2 \mathrm{H}, J=$ 4.2, $13.0 \mathrm{~Hz}, \mathrm{H}-7$ ' $\beta, \mathrm{H}-7$ ' $\beta$ ), 3.26 (dd, $1 \mathrm{H}, J=6.0,9.2 \mathrm{~Hz}, \mathrm{H}-1 \alpha), 3.68(\mathrm{~d}, 1 \mathrm{H}$, $J=5.4,9.2 \mathrm{~Hz}, \mathrm{H}-1 \beta), 4.43$ (s, $2 \mathrm{H}, \mathrm{OH}), 5.94\left(\mathrm{~s}, 2 \mathrm{H}, \mathrm{OCH}_{2} \mathrm{O}\right), 5.98(\mathrm{~s}, 2 \mathrm{H}$, $\left.\mathrm{OCH}_{2} \mathrm{O}\right), 6.64-6.78(\mathrm{~m}, 6 \mathrm{H}, \mathrm{ArH}) .{ }^{13} \mathrm{C}$ NMR $\left(75 \mathrm{MHz}, \mathrm{CDCl}_{3}\right): \delta 34.8(\mathrm{C}-7$ ', $\mathrm{C}-7$ ', $), 43.9(\mathrm{C}-2, \mathrm{C}-3), 65.2(\mathrm{C}-1, \mathrm{C}-4), 100.6\left(2 \times \mathrm{OCH}_{2} \mathrm{O}\right), 107.9(\mathrm{C}-5$ ', C-5'), 108.9 (C-2', C-2'”), 133.2 (C-6', C-6"), 135.0 (C-1', C-1' '), 145.2 (C4', C-4"'), 146.7 (C-3', C-3"). EI-MS (m/z, \%): $356\left(\mathrm{M}^{+}, 40\right), 267$ (23), 254 (11), 200 (16), 151 (100). HRMS calcd for $\mathrm{C}_{20} \mathrm{H}_{24} \mathrm{NO}_{6}\left(\mathrm{M}+\mathrm{NH}_{4}^{+}\right): 374.1599$. Found: 374.1605. Anal. Calcd for $\mathrm{C}_{24} \mathrm{H}_{35} \mathrm{O}_{6}$ : $\mathrm{C}, 68.71 ; \mathrm{H}, 8.41$. Found: $\mathrm{C}$, $68.65 ; \mathrm{H}, 8.37$. The spectral data were in agreement with the literature ${ }^{21}$.

\section{RESULTS AND DISCUSSION}

\section{Synthesis of compounds}

Piperonal 1a or veratraldehyde $\mathbf{1 b}$ was reacted with diethyl succinate in sodium ethoxide-ethanol solution to produce the benzylidene succinate halfester, following by the esterification provided the diester $\mathbf{2 a}$ or $\mathbf{2 b}$. Alkylation reaction of $\mathbf{2} \mathbf{a}$ or $\mathbf{2} \mathbf{b}$ to diester $\mathbf{3} \mathbf{a}$ or $\mathbf{3 b}$ was achieved by treatment with LDA and 3, 4-methylenedioxybenzyl bromide under $-78^{\circ} \mathrm{C}$. Hydrolysis of diester 3a or $\mathbf{3} \mathbf{b}$ under conventional conditions $\left(\mathrm{NaOH}, \mathrm{H}_{2} \mathrm{O}\right)$ produced diacid, following by resolution via the quinine salt afforded diacid (-)-4a or (-)-4b, (+)-4a' or $(+)-\mathbf{4 b}$ '. The diacid $(-)-\mathbf{4 a}$ or $(-)-\mathbf{4 b}$ was esterified to produce unsaturated diester $(-)-\mathbf{5 a}$ or $(-)-\mathbf{5 b}$ (Scheme 1).
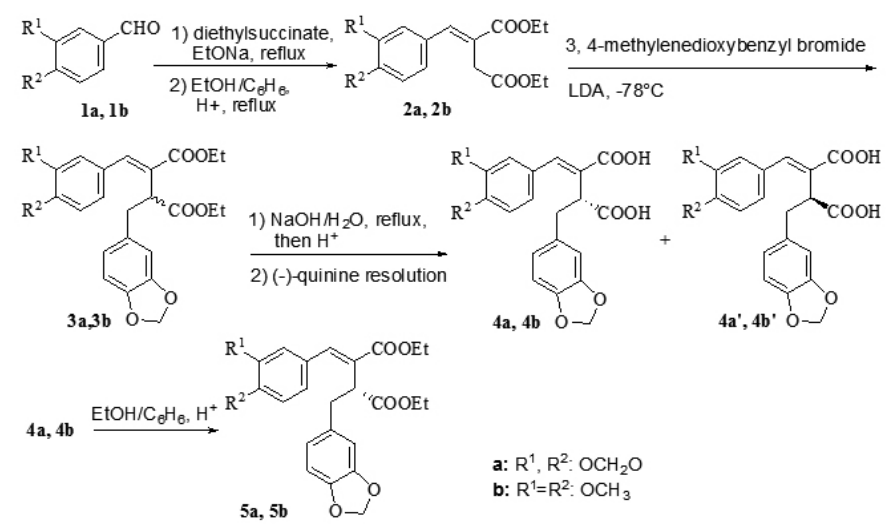

Scheme 1 Synthesis of compounds $\mathbf{5 a}, \mathbf{5 b}$

Reaction of (-)-5a or (-)-5b with $\mathrm{LiAlH}_{4} / \mathrm{AlCl}_{3}$ in THF afforded unsaturated diol lignans (-)-6a or (-)-6b (Scheme 2).

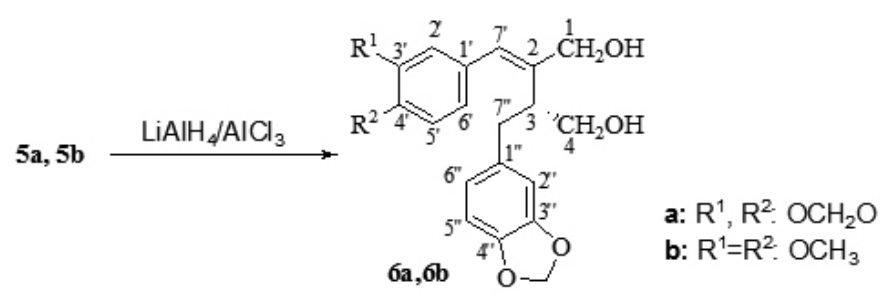

Scheme 2 Synthesis of compounds $\mathbf{6 a}, \mathbf{6 b}$

Treatment of (-)-5a or (-)-5b with $\mathrm{Pd} / \mathrm{C}(10 \%)$ under hydrogen atmosphere gave saturated diester, following by reduction with $\mathrm{LiAlH}_{4}$ in THF to produce a readily separable mixture (approximate 1:1) of dibenzylbutanediol lignans threo-(-)-7a and erythro-8a or threo-(-)-7b and erythro-8b (Scheme $3)$. erythro-7a did not have optical rotation and was a meso-compound. The spectral data threo-(-)-7a, erythro-8a and threo-(-)-7b were in agreement with those found in the literatures.

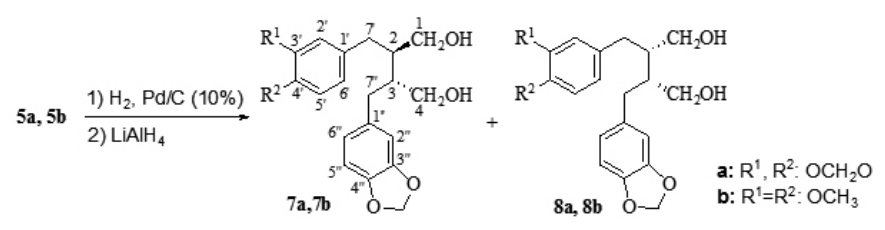

Scheme 3 Synthesis of compounds 7a, 7b, 8a, 8b

When (-)-5a was treated with DDQ in acetic acid dehydrogenation occurred to give the compound $\mathbf{9}$. Treatment of (-)-5a with DDQ in trifluoroacetic acid dehydrogenation afforded the dibenzocyclooctadiene diol 10 (Scheme 4). 


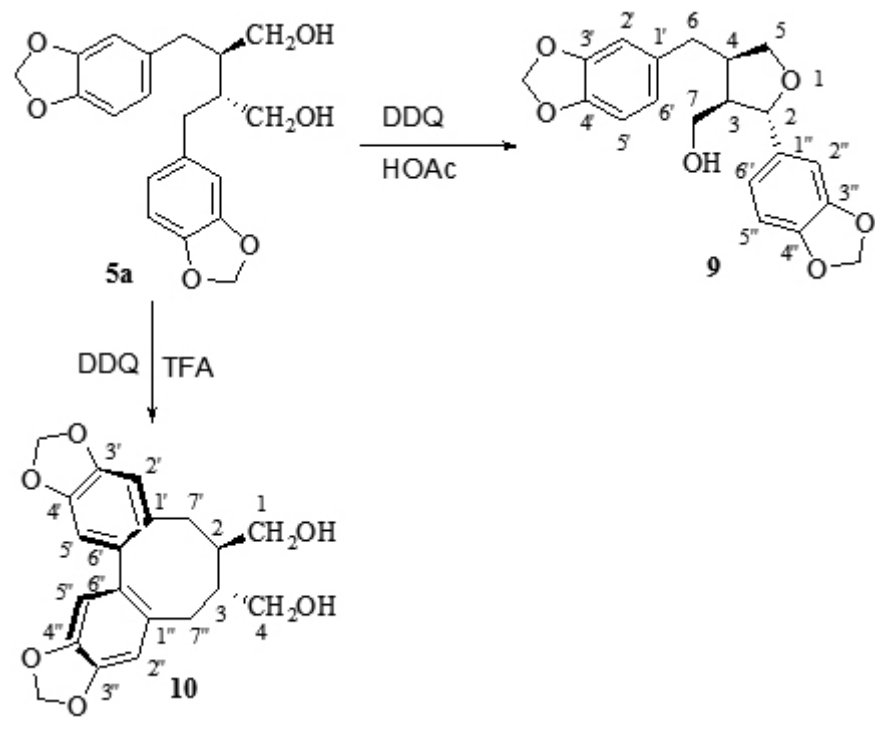

Scheme 4 Synthesis of compounds $\mathbf{9 ,}, \mathbf{1 0}$

\section{Bioactivity}

Anti-virus activity

The synthesized compounds $\mathbf{6 a}, \mathbf{6 b}, 7 \mathbf{a}, 7 \mathbf{b}, \mathbf{8 a}, \mathbf{8 b}, 9$ and 10 were evaluated for their antiviral activities against HIV-1 and HSV in vitro. The anti-HIV assays of the synthesized lignans reported here were in agree with the methods previously reported ${ }^{22}$.

Among all the synthesized compounds, derivatives $\mathbf{7 a}, \mathbf{7 b}, \mathbf{8 a}, \mathbf{8 b}$ with two free hydroxyl group were the most potent inhibitors of HIV replication, coupled with the highest selectivity index. In fact, compound erythro-8b showed the weak activities against HIV-1 $\left(\mathrm{IC}_{50}=150 \mu \mathrm{g} / \mathrm{ml}\right)$, while the rest of the compounds did not exhibit any obvious activity.

The process of anti-HSV assay was previously described ${ }^{23}$. The results showed that $\mathbf{8 b}$ exhibited antiviral activity against herpes virus $(77.04 \mu \mathrm{g} / \mathrm{ml})$. The rest of the compounds did not exhibit any obvious activity. On the other hand, the results showed that SI of $\mathbf{8 b}$ was much lower $(\mathrm{SI}<1.0)$. Thus, we should enhance SI in the latter study in structure-function relationship in order to increase the selectivity of activity.

The biological data of the synthesized compounds led to some considerations that permitted a more regular pattern. Only compound $\mathbf{8 b}$, bearing two free hydroxyl groups with erythro-structure, was characterized by modest antiviral activity against both HIV-1 and HSV. Thus, within the dibenzylbutanediol lignans, the best activity was provided by the two free hydroxyl groups and erythro-structure.

\section{Anti-tumor activity}

The synthesized dibenzylbutanediol lignans and their analogues were evaluated against HL-60 human leukemic cell, PC-3MIE8 human prostatic carcinoma cell, BGC-823 human stomach cancer cell, MDA-MB-435 human breast cancer cell in vitro, and the assays of the lignans have been previously published ${ }^{24}$

We observed that all of the compounds at $10^{-5} \mathrm{M}, 10^{-6} \mathrm{M}$ and $10^{-7} \mathrm{M}$ inhibited HL-60 cell, PC-3MIE8 cell, BGC-823 cell, Bel-7402 cell and MDA-MB-435 cell. Most interesting activities, however, were showed against MDA-MB-435 human breast cancer cell. In a screen against MDA-MB-435 human breast cancer cell, we observed that the compound $\mathbf{1 0}$ possessed weak activity and showed inhibition ratio above $22.3 \%$ at $10^{-5} \mathrm{M}$. The anti-tumor activities of the other compounds indicated the inhibitory rates of tumor cell were less than $20 \%$ or showed no obvious anti-tumor activity.

The biological activity of the synthesized compounds showed the dibenzylbutanediol lignans exhibited low inhibition ratio against tumor cell. Among these serial modifications, only diol-10 with dibenzocyclooctadiene structure gave the weak activity against human breast cancer cell. Thus, dibenzocyclooctadiene structure seems to be beneficial to inhibit breast cancer cell and led to an increase in anti-tumor activity.

\section{CONCLUSIONS}

In the aspect of synthesis of compounds, we have developed an efficient chiral synthetic route to give eight dibenzylbutanediol lignans including four natural products. With cheap materials, short experimental procedures, mild conditions and simple operations, the route exhibited more potential value in the future. Within the dibenzylbutanediol lignans, lignan $\mathbf{8 b}$ with the two free hydroxyl groups and erythro-structure showed the best anti-virus activity. In the aspect of anti-tumor activity, lignan $\mathbf{1 0}$ with dibenzocyclooctadiene structure seem to be beneficial to inhibit breast cancer cell and dibenzocyclooctadiene ring led to an increase in anti-tumor activity.

\section{ACKNOWLEDGEMENTS}

This work was supported by Taishan Scholar Project of Shandong Province (No. 2006011036) and Open Foundation of Chemical Engineering Subject, Qingdao University of Science \& Technology.

\section{REFERENCES}

1. R. S. Ward, Nat. Prod. Rep. 16, 75, (1999)

2. M. Saleem, H. J. Kim, M. S. Ali, Y. S. Lee, Nat. Prod. Rep. 22, 696 , (2005)

3. J. Chang, J. Reiner, J. X. Xie, Chem. Rev. 105, 4581, (2005)

4. R. T. Lalonde, F. Ramdayal, A. Sarko, K. Yanai, M. J. Zhang, J. Med. Chem. 46, 1180, (2003)

5. N. Li, J. Wu, T. Hasegawa, J. Sakai, L. Bai, L. Wang, S. Kakuta, Y. Furuya, H. Ogura, T. Kataoka, A. Tomida, T. Tsuruo, M. Ando, Nat. Prod. 70, 544, (2007)

6. Y. W. Chin, H. B. Chai, W. J. Keller, A. D. Kinghorn, Agric. Food. Chem. 56, 7759, (2008)

7. P. J. Magee, H. Mcglynn, I. R. Rowland, Cancer Lett. 208, 35, (2004)

8. S. K. Chattopadhyay, T. R. S. Kumar, P. R. Maulik, S. Srivastava, A. Garg, A. Sharon, A. S. Negi, S. P. S. Khanuja, Bioorg. Med. Chem. 11, 4945, (2003)

9. E. Kupeli, N. Erdemoglu, E. Yesilada, B. Sener, J. Ethnopharmacol. 89, $265,(2003)$

10. W. D. Macrae, G. H. N. Towers, Phytochemistry 23, 1207, (1984)

11. T. Nakato, S.Yamauchi, J. Nat. Prod. 70, 544, (2007)

12. S. M. Miles, S. P. Marsden, R. J. Leatherbarrow, W. J. Coates, J. Org. Chem. 69, 6874, (2004)

13. B. Raffaelli, K. Wahala, T. Hase, Org. Biomol. Chem. 4, 331, (2006)

14. P. Eklund, A. Lindholm, J. P. Mikkola, A. Smeds, R. Lehtila, R. Sjoholm, Org. Lett. 5, 491, (2003)

15. Q. Wang, Y. Yang, Y. Li, W. Yu, Z. J. Hou, Tetrahedron 62, 6107, (2006)

16. A. Pelter, R. S. Ward, D. M. Jones, P. Maddocks, Tetrahedron Asymmetry 3, 239, (1992)

17. A. S. R. Anjaneyulu, P. A. Ramaiah, L. R. Row, R. Venkateswarlu, A. Pelter, R. S. Ward, Tetrahedron 37, 3641, (1981)

18. M. J. Kato, M. Yoshida, O. R. Gottlieb, Phytochemistry 31, 283, (1992)

19. P. Satyanarayana, S. Venkateswarlu, Tetrahedron 47, 8931, (1991)

20. S. C. Roy, K. K. Rana, C. Guin, J. Org. Chem. 67, 3242, (2002)

21. A. Pelter, R. S. Ward, R. Venkateswarlu, C. Kamakshi, Tetrahedron 47, $1275,(1991)$

22. G. T. Tan, J. M. Pezzuto, A. D. Kinghorn, S. H. Hughes, J. Nat. Prod. 54 $143,(1991)$

23. R. Dulbeccok, Proc. Natl. Acad. Sci. U.S.A. 38, 741, (1952)

24. J. L. Wu, N. Li, T. Hasegawa, J. Sakai, S. Kakuta, W. X. Tang, S. Oka, M. Kiuchi, H. Ogura, T. Kataoka, A. Tomida, T. Tsuruo, M. Ando, J. Nat. Prod. 68, 1656, (2005) 